\title{
SHORT COMMUNICATION Cell cycle-dependent acetylation of Rb2/p130 in NIH3T3 cells
}

\author{
F Schwarze ${ }^{1,4}$, J Meraner ${ }^{1,4}$, M Lechner ${ }^{1}$, A Loidl ${ }^{1}$, T Stasyk ${ }^{2}$, A Laich ${ }^{3}$ and P Loidl ${ }^{1}$ \\ ${ }^{1}$ Division of Molecular Biology, Biocenter, Innsbruck Medical University, Innsbruck, Austria; ${ }^{2}$ Division of Cell Biology, Biocenter, \\ Innsbruck Medical University, Innsbruck, Austria and ${ }^{3}$ Tyrolean Cancer Research Institute, Innsbruck, Austria
}

\begin{abstract}
The retinoblastoma protein $(\mathrm{pRb})$ and the $\mathrm{pRb}$-related proteins, p130 and p107, form the 'pocket protein' family of cell cycle regulatory factors. A well characterized function of these proteins is the cell cycle-dependent regulation of E2F-responsive genes. The biological activity of pocket proteins is regulated by phosphorylation and for the founding member $\mathrm{pRb}$ it has been shown that acetylation also has an important role in modulating its function during the cell cycle. Here, we show that hyperphosphorylated retinoblastoma 2 (Rb2)/p130 also exists in an acetylated form in NIH3T3 cells. Acetylated p130 is present in the nucleus but not in the cytoplasm. Acetylation is cell cycle dependent, starting in S-phase and persisting until late $\mathbf{G}_{2}$-period. Using recombinant p130 and truncated forms for in vitro acetylation by the acetyltransferase p300, we could identify $K 1079$ in the C-terminal part as the major acetylation site by mass spectrometry. Minor acetylation sites were pinpointed to K1068 and K1111 in the C-terminus, and K128 and K130 in the $\mathrm{N}$-terminus. The human papilloma virus 16 proteinE7 preferentially binds to acetylated p130 and significantly increases in vitro p130 acetylation by p300.

Oncogene (2010) 29, 5755-5760; doi:10.1038/onc.2010.311; published online 2 August 2010
\end{abstract}

Keywords: Rb2/p130; cell cycle; acetylation; p300; posttranslational modification

The retinoblastoma gene family consists of three members, retinoblastoma $(\mathrm{Rb}) / \mathrm{p} 105, \mathrm{p} 107$ and $\mathrm{Rb} 2 /$ p130. The family is also referred to as the "pocket protein' family, as these proteins are characterized by a conserved pocket region through which they can bind viral and cellular proteins, such as the E2F family of transcription factors (Claudio et al., 2002; Du and Pogoriler, 2006). The $\mathrm{Rb}$ family proteins have important roles in proliferation control by transcriptional regulation of cell cycle progression through E2F, especially at the $\mathrm{G}_{1}-\mathrm{S}$ transition (Cobrinik, 2005); moreover, pocket proteins can target chromatin modifiers to E2F promo-

Correspondence: Dr P Loidl, Division of Molecular Biology, Biocenter, Innsbruck Medical University, Fritz-Pregl-Strasse 3, Innsbruck 6020, Austria.

E-mail: Peter.Loidl@i-med.ac.at

${ }^{4}$ These authors contributed equally to this work.

Received 15 April 2009; revised 29 April 2010; accepted 1 June 2010; published online 2 August 2010 ters and interact with chromatin remodeling enzymes (Giacinti and Giordano, 2006; Macaluso et al., 2006).

Chromatin modifying enzymes not only modify histones, but indeed a huge variety of non-histone regulatory and structural proteins that interact with chromatin, such as protooncogene and tumor suppressor proteins, transcription factors and enzymes (Gu and Roeder, 1997; Vervoorts et al., 2003; Zhang et al., 2003; Choi et al., 2003; Meraner et al., 2006). Retinoblastoma protein $(\mathrm{pRb})$ has been shown to be acetylated by $\mathrm{p} 300 /$ CBP, as well as P/CAF (Nguyen et al., 2004) and the modification changes its properties with respect to phosphorylation by cyclin-dependent kinases (Chan et al., 2001).

The cell cycle-dependent phosphorylation of more than 20 distinct residues has been identified for $\mathrm{Rb} 2 /$ p130 (Canhoto et al., 2000; Hansen et al., 1999, 2001). The majority of sites can be phosphorylated by CDK-2, 4 or 6 , predisposing p130 for ubiquitination, and thus proteosomal degradation (Tedesco et al., 2002). However, in contrast to the common picture of pocket protein inactivation through phosphorylation by CDKs, p130 actively associates with E2F-4 in a distinct phosphorylation state as cells enter $\mathrm{G}_{0}$ (Mayol et al., 1996); this modification state is independent from CDK activity and has been ascribed to glycogen synthase kinase 3 (Litovchick et al., 2004). Moreover, mapping of phosphorylation sites revealed only 3 out of 22 CDK consensus sites being conserved between pRB and p130, whereas 10 phosphorylated serine/threonine residues are conserved between p107 and p130, indicating pronounced differences in the functional consequences of modification among the three pocket proteins.

We set out to study the posttranslational acetylation of p130 during the cell cycle of NIH3T3 cells, as a screening for acetylated non-histone nuclear proteins revealed p130 to be modified by acetylation. NIH3T3 cells were synchronized in $\mathrm{G}_{0}$ by serum starvation for $72 \mathrm{~h}$. After stimulation through serum addition cells were harvested at $2 \mathrm{~h}$ intervals for a total of $24 \mathrm{~h}$. Nuclear extracts were then prepared and analyzed by immunoblotting with monclonal antibodies against p130 (BD Transduction Laboratories, San Jose, CA, USA) and acetylated lysine. Serum-starved cells in $\mathrm{G}_{0}$ showed substantial amounts of hypo-phosphorylated p130 and low amounts of the slower migrating hyperphosphorylated form of p130. During the time course of the experiment the abundance of hypophosphorylated p130 continuously decreased, whereas the amount of the hyperphosphorylated form increased 
significantly until $6-8 \mathrm{~h}$ after serum addition and stayed on a high level until $\mathrm{G}_{2}$-period (Figure 1a). A corresponding cell cycle pattern of hypo- and hyperphosphorylated forms was also obtained when polyclonal p130 antibodies (Santa Cruz Biotechnology, Santa Cruz, CA, USA) were used for immunoblotting. This pattern of hypo- and hyper-phosphorylated p130 has been previously reported for a time period of $12 \mathrm{~h}$ after serum addition in T98G cells by Hansen et al. (2001). In $G_{0}$ and $G_{1}$ immunoblotting with anti-acetyl lysine antibodies did not yield an immunosignal with either hypo- or hyper-phosphorylated p130. However, in early S-phase the hyperphosphorylated form of p130 shifted into the acetylated form as anti-acetyl lysine antibodies yielded an immunosignal that co-migrated with hyperphosphorylated p130; this band persisted until late $\mathrm{G}_{2}$-period (Figure 1a). This result was confirmed with a specific antibody $(\alpha-\mathrm{K}-1079 \mathrm{ac})$ that we raised against a C-terminal p130 peptide from amino acids $1074-1085$ containing $\mathrm{K}-1079$ in the acetylated state (SNSPSK ${ }_{\mathrm{ac}}$ RLREIN; see results below). The cell cycle distribution of cells was monitored by immuno- detection of cyclin B1 (Figure 1a) and fluorescenceactivated cell sorting analysis (not shown). These results suggest that a significant proportion of p130 in a hyperphosphorylated state is acetylated in early S-phase and is present in this state until late $\mathrm{G}_{2}$-period.

In order to further substantiate that hyperphosphorylated p130 is acetylated in S-phase, we performed small interfering RNA knockdown of p130 and analyzed $\mathrm{S}$-phase nuclear extracts at $16 \mathrm{~h}$ after serum addition by immunoblotting using antibodies against p130, acetylated lysine residues and the specific antibody against p130 acetylated at K-1079. The immunoblot with antip130 antibodies clearly shows that the hypo- and hyperphosphorylated protein bands significantly decreased in si-p130 transfected cells; in line with this, the immunoblot with the antibody against the acetylated p130 peptide $1074-1085(\alpha-\mathrm{K}-1079 \mathrm{ac})$ showed a significant decrease of the slower migrating, hyperphosphorylated p130 form (Figure 1b). A corresponding result was obtained with anti-acetyl lysine antibodies; a glyceraldehyde 3-phosphate dehydrogenase knockdown control corroborated the results (Figure 1b).
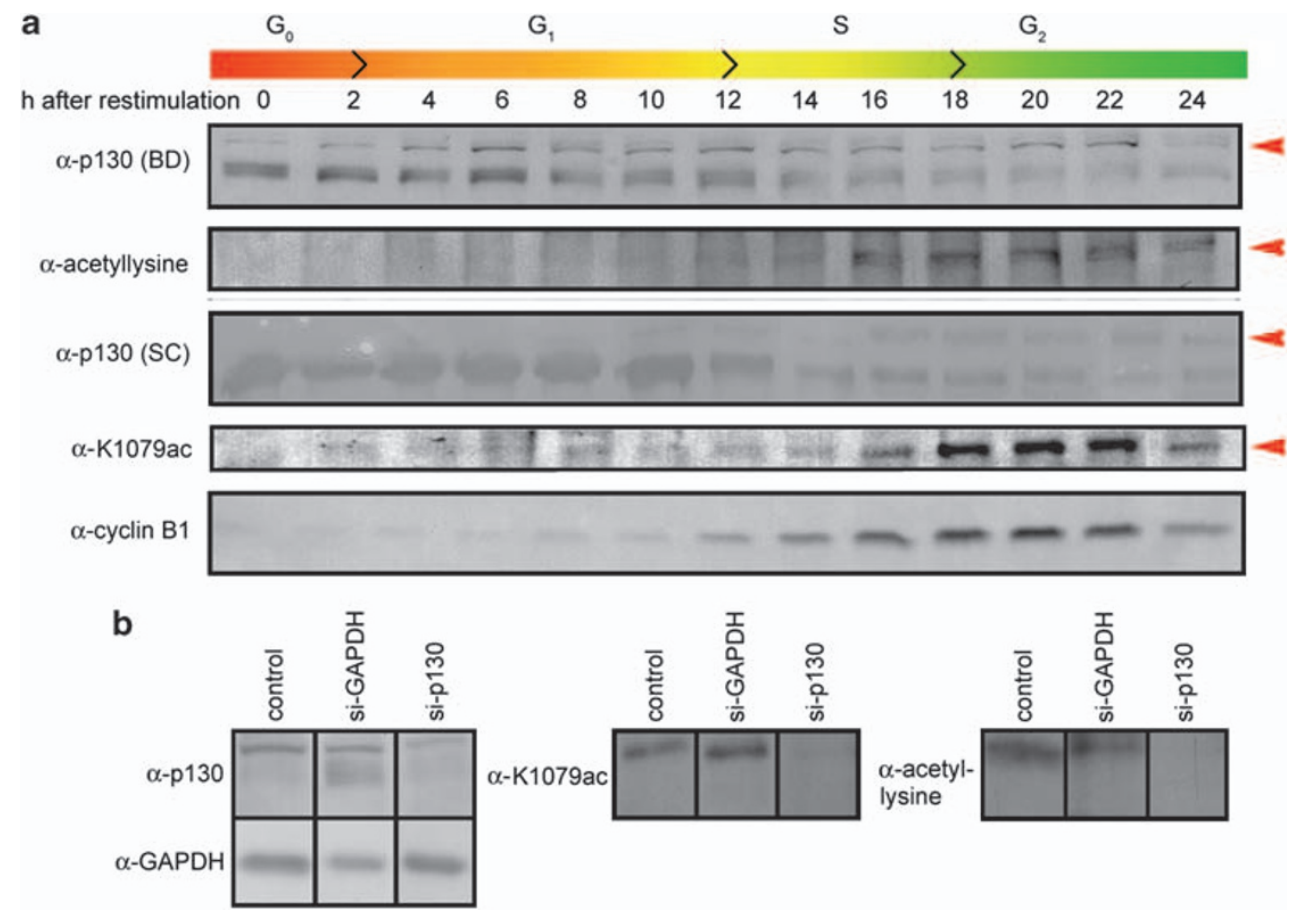

Figure 1 Cell cycle dependent abundance of p130 forms in NIH3T3 cells. (a) NIH3T3 cells were serum starved for 72 h. After serum addition cells were harvested at $2 \mathrm{~h}$ intervals. Nuclei were isolated and nuclear extracts were prepared using NE-PER Nuclear and Cytoplasmic Extraction Reagents (Pierce, Thermo Fisher Scientific, Rockford, IL, USA). Equal amounts of protein from each sample were subjected to SDS- $10 \%$ polyacrylamide gel electrophoresis (SDS-10\% PAGE) with subsequent blotting to nitrocellulose. p130 was detected either with a monoclonal antibody (BD; BD Transduction Laboratories; No.610262) or with polyclonal antibodies (SC; Santa Cruz, sc-317). Acetyl lysine was detected with a polyclonal antibody (Upstate, Millipore-Merck, Darmstadt, Germany; No.06933), acetylated p130 with a specific antibody that was raised in rabbits against a C-terminal p130 peptide (aa 1074-1085) where K-1079 is acetylated $(\alpha-\mathrm{K}-1079 \mathrm{ac})$, and cyclin B1 with a monoclonal antibody (Santa Cruz; sc-245). Arrows highlight the hyperphosphorylated, acetylated p130 forms. (b) Effect of si-p130 knockdown in synchronized S-phase cells at $16 \mathrm{~h}$ after serum addition. Small interfering RNA (siRNA) was purchased from Dharmacon (Lafayette, CA, USA; RBL2-p130-mouse siGENOME-ON-TARGET plus set of four, glyceraldehyde 3-phosphate dehydrogenase (GAPDH)-mouse control) and resuspended in DEPC treated PBS. NIH$3 \mathrm{~T} 3$ cells were grown to $90 \%$ confluency, and transfected with a mix of 4 RBL2/p130 siRNAs (100 nm each) or GAPDH siRNA and metafectene (ratio 1:4) according to the manufacturer's instructions in serum free medium. Cells were serum starved for $72 \mathrm{~h}$, before stimulation with $10 \%$ fetal calf serum to re-enter the cell cycle for $16 \mathrm{~h}$. Cells were then harvested, washed and nuclear extracts were prepared using NE-PER. Proteins were separated by SDS-10\% PAGE, blotted onto nitrocellulose and immunodetected using the indicated antibodies. Bands were visualized using electrochemiluminescence. 
In order to further prove acetylation of hyperphosphorylated p130, we performed immunoprecipitation using anti-p130 antibodies for precipitation of nuclear extracts from NIH3T3 cells in $\mathrm{G}_{0}$ and S-phase. After electrophoresis, immunoblotting was done with antibodies against p130, acetyl lysine, and the acetylated C-terminal p130 peptide $(\alpha-\mathrm{K}-1079 \mathrm{ac})$. Figure 2a shows that anti-p130 antibodies precipitate p130 with the hyperphosphorylated form being acetylated, as detected by anti-acetyl lysine, as well as specific anti-p130K-1079ac antibodies. To get an idea of a possible intracellular localization of acetylated p130, we analyzed nuclear and cytoplasmic extracts from exponentially growing NIH3T3 cells. Figure 2b shows that anti-acetyl lysine antibodies detected acetylated p130 exclusively in nuclear extracts, although the amount of hyperphosphorylated p130 is higher in the cytoplasmic extract. To corroborate this result, we again used siRNA knockdown of p130 in exponentially growing NIH3T3 cells (Figure 2c). Immunoblotting of nuclear and cytoplasmic extracts of si-p130 transfected cells revealed efficient downregulation of p130 in both forms (hypo- and hyper-phosphorylated), whereas an small interferingglyceraldehyde 3-phosphate dehydrogenase control yielded the localization pattern as seen in the untransfected wild type (Figures 2b and c). As expected, immunoblotting with the specific antibody against acetylated p130 ( $\alpha-\mathrm{K}-1079 \mathrm{ac})$ showed the acetylated and hyperphosphorylated p130 in nuclear extracts of the small interfering-glyceraldehyde 3-phosphate dehydrogenase control, but neither in the cytoplasmic extract of the small interfering-glyceraldehyde 3-phosphate dehydrogenase control nor in the nuclear extracts of si-p130 knockdown cells (Figure 2c). From these results, we conclude that acetylated, hyperphosphorylated p130 is exclusively located in the nucleus, although the main proportion of hyperphosphorylated p130 resides in the cytoplasm (in a non-acetylated state).

As the total amount of p130 is very low in NIH3T3 cells, we expressed N-terminally FLAG-tagged p130 (Figure 3a) in insect cells and used the recombinant protein for in vitro acetylation by the acetyltransferase p300. Using radioactive acetyl-CoA as a co-substrate, electrophoretic analysis and subsequent autoradiography of the assay product revealed that p130 is acetylated by p300 in vitro (Figure 3b; lane 4, autoradiography panel). Corresponding assays were performed with non-radioactive acetyl-CoA and acetylated p130, as well as non-acetylated p130 were subsequently analyzed by mass spectrometry. This revealed a dominant acetylation site at K-1079 (Supplementary Information; Figure 1) and minor acetylation at $\mathrm{K}-1068$ and $\mathrm{K}-1111$ in the C-terminus and at K-128 and $\mathrm{K}-130$ in the N-terminus. To substantiate these findings, we expressed additional truncated p130 forms in insect cells (Figure 3a): FLAG-tagged versions
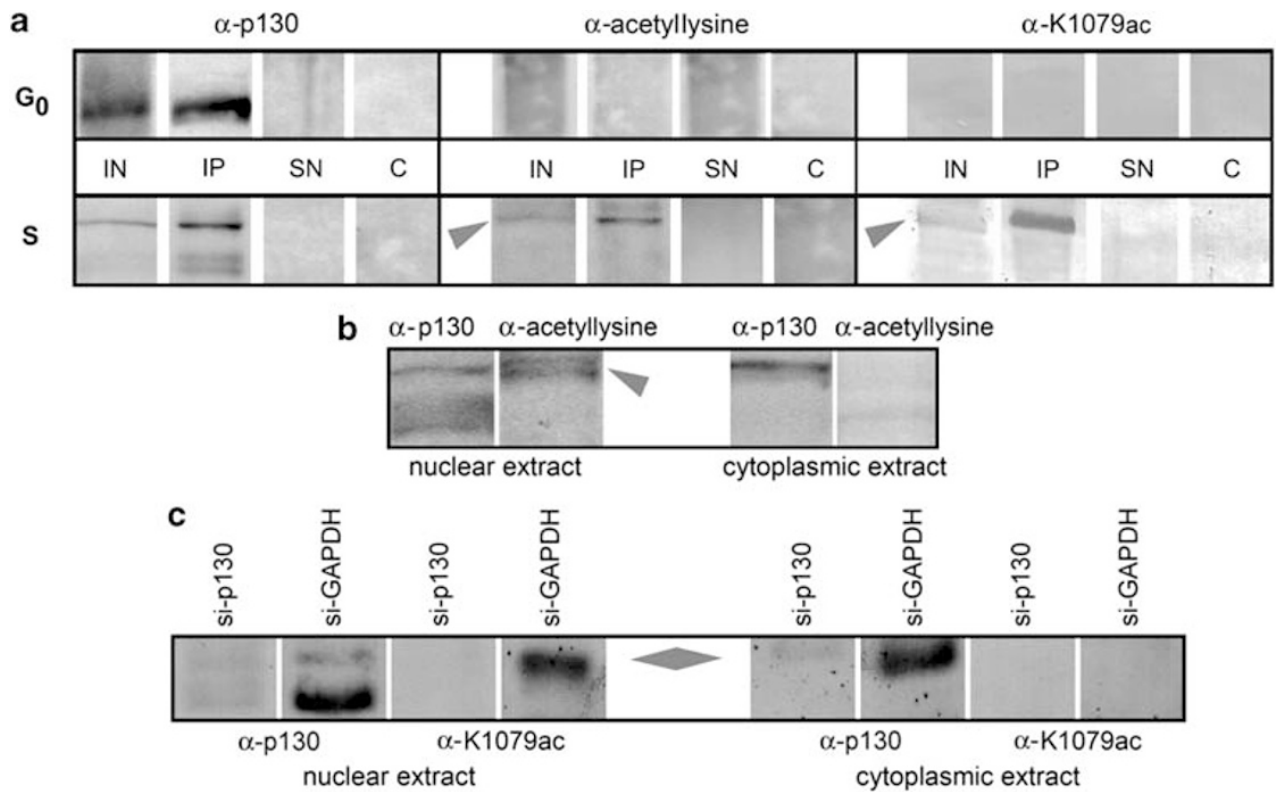

Figure 2 p130 from nuclear extracts exists in an acetylated form. (a) Nuclear extracts from synchronized NIH3T3 cells in $\mathrm{G}_{0}$-phase $(0 \mathrm{~h}$ after serum addition) or S-phase ( $16 \mathrm{~h}$ after serum addition) were immunoprecipitated with antibodies against p130 (BD Transduction Laboratories) coupled to Protein-G-Sepharose beads. After washing, beads (IP), supernatant (SN), as well as nuclear extract (input, IN) were subjected to SDS-10\% polyacrylamide gel electrophoresis (SDS-10\% PAGE) with subsequent immunoblotting using antibodies against p130 (BD), acetyl lysine or K-1079ac, respectively. As an immunoglobulinG control, nuclear extract lanes were also incubated with secondary antibodies alone (c, control). Proteins were visualized with alkaline phosphatase conjugated secondary antibodies. (b) Exponentially growing NIH3T3 cells were fractionated into nuclear and cytoplasmic extracts. After SDS-10\% PAGE and blotting onto nitrocellulose, p130 and acetylated p130 were detected by the corresponding antibodies. (c) Exponentially growing NIH3T3 cells were transfected with si-p130 or small interfering-glyceraldehyde 3-phosphate dehydrogenase (si-GAPDH) for $72 \mathrm{~h}$ as described in the legend of Figure 1. After harvesting, cells were fractionated and samples were processed and analysed as described above. Arrows highlight the position of acetylated p130. 


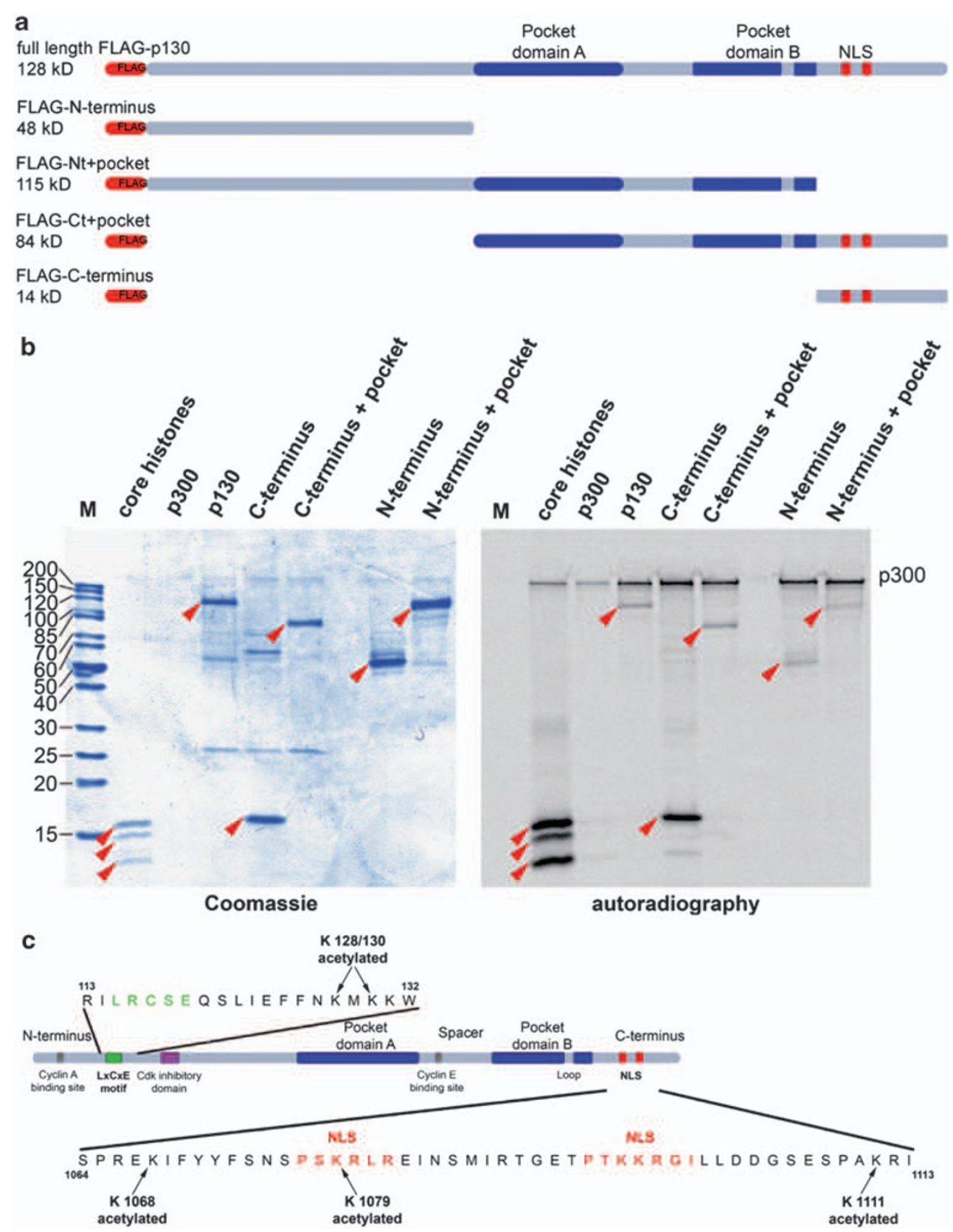

Figure 3 Acetylation of $\mathrm{p} 130$ by $\mathrm{p} 300$ acetyltransferase in vitro takes place predominantly in the C-terminus and the major acetylation site can be pinpointed to K-1079. (a) Schematic representation of N-terminally FLAG-tagged full-length p130, as well as truncated versions expressed in Sf9 cells (baculovirus system). (b) Full-length and truncated versions of p130 were used for in vitro acetylation assays. Proteins were incubated with $\mathrm{p} 300$ and $20 \mu \mathrm{M}{ }^{14} \mathrm{C}$-acetylCoA for $30 \mathrm{~min}$ at $37^{\circ} \mathrm{C}$, visualized by Coomassie blue stain (left panel) and autoradiography (right panel) after SDS-10\% polyacrylamide gel electrophoresis (SDS-10\% PAGE). Histones and p300 were used as controls. (c) Lysine residues found to be acetylated by MALDI-TOF/TOF mass spectrometry are shown in their respective sequence environment. Ct, C-terminus; NLS, nuclear localization sequence; Nt, N-terminus.

of the $\mathrm{N}$-terminus ( $48 \mathrm{kDa})$, $\mathrm{N}$-terminus + pocket domain $(115 \mathrm{kDa}), \mathrm{C}$-terminus + pocket domain $(84 \mathrm{kDa})$ and C-terminus $(14 \mathrm{kDa})$. We used these proteins for radioactive in vitro assays with p300 and subsequently analyzed them by SDS-polyacrylamide gel electrophoresis and autoradiography. Figure $3 \mathrm{~b}$ shows that the five recombinant proteins were produced in sufficient quantity and quality (Coomassie panel) and approximately equal amounts were used for the p300-histone acetyltransferase assay. As control we used chicken erythrocyte histones. Autoradiography revealed that full-length p130 and C-terminus + pocket domain construct were labeled roughly equally, whereas the isolated C-terminus exhibited particularly high acetylation by p300 (Figure 3b, autoradiography panel). The truncated p130 versions comprising the $\mathrm{N}$-terminus and the $\mathrm{N}$-terminus + pocket were acetylated to a lesser extent. As expected, autoacetylation of p300 and prominent acetylation of core histones are visible. Note, that no unspecific acetylation of contaminating minor proteins 
could be detected, emphasizing the specificity of p130 acetylation.

The fact that the $\mathrm{C}$-terminus and the constructs that contain the $\mathrm{C}$-terminus are acetylated stronger than the $\mathrm{N}$-terminal constructs is in line with mass spectrometry results that clearly identified a major acetylation site at K-1079, whereas the other acetylated residues represented minor sites. Acetylated sites are depicted in Figure 3c. The major acetylatable K-1079 is located within one of two C-terminal nuclear localization sequences, implying a possible effect on subcellular localization of $\mathrm{p} 130$. Therefore, we performed transient transfection experiments with C-terminal p130/GFP constructs where K-1079 was mutated to arginine or glutamine, but could not observe a significant change of nuclear localization of the reporter protein by microscopy of transfected cells (results not shown), indicating that a more subtle mechanism is modulating nuclear localization of p130 rather than a simple K-1079 acetylation. This is in line with previous results (Chestukhin et al., 2002), showing that functional deletion of one of the two nuclear localization sequences alone was not sufficient to abolish nuclear localization of p130, whereas only deletion of both resulted in reduced nuclear localization (Chestukhin et al., 2002). In this respect, it is interesting that all three acetylated lysines in the C-terminus (K-1068, K-1079 and K-1111) are in close neighborhood (3 or 4 amino acids) to documented phosphorylation sites (S-1064, S-1076 and S-1108; Hansen et al., 2001); in all three cases the phosphorylated serine is followed by a proline. It is tempting to speculate that the prolines might be substrates of peptidyl-proline isomerases, which would induce secondary structure changes. It has been shown that a proline isomerase acts on prolines 30 and 38 of histone $\mathrm{H} 3$, thereby modulating methylation of a neighboring lysine (Nelson et al., 2006). An interrelation between proline isomerization and p300 recruitment has been demonstrated for $\mathrm{p} 73$ (Mantovani et al., 2004). The fact of phosphorylation and acetylation sites together with proline residues being clustered within few amino acids, and all clusters being located within a region of only 50 amino acids, suggests that the C-terminus of p130 may be a region of particular regulatory potential in the sense of a protein modification code (Brosch et al., 2008; Sims and Reinberg, 2008).
It has been shown that $\mathrm{p} 130$ is predominantly abundant in $\mathrm{G}_{0^{-}}$and $\mathrm{G}_{1}$-phases (Claudio et al., 2002) and rapidly decreases after S-phase (Classon and Dyson, 2001); in NIH3T3 cells we did not observe such a rapid decrease of p130. The modified forms of nuclear p130 rather persisted through $\mathrm{G}_{2}$-period (Figure 1), a finding consistent with a role for p130 in active $\mathrm{G}_{2}$-complexes (Popov et al., 2005) and in cell cycle exit in $\mathrm{G}_{2}$ (Jackson et al., 2005).

To demonstrate a functional significance of acetylation for the properties of p130, we analyzed p300mediated acetylation of p130 in the presence of the human papilloma virus16 protein-E7 (HPV16-E7). Pocket proteins have been shown to be targets of small DNA virus oncoproteins, such as proteins from certain types of papilloma-, polyoma- and adeno-viruses (Felsani et al., 2006; White and Khalili, 2006). HPV16E7 binds to p130 and targets it for degradation; moreover, histone deacetylases bind to p130 and the E7 protein, thereby inhibiting histone deacetylase binding to the E2F2 promoter and elevating the acetylation of histones associated with E2F-responsive promoters (Roman, 2006). We used full-length p130 protein, the C-terminus + pocket construct and the $\mathrm{N}$-terminus for radioactive in vitro acetylation by $\mathrm{p} 300$ in the presence or absence of HPV16-E7. Figure 4 shows that E7 protein enhances p300 mediated acetylation of full-length p130; this enhancement was even more pronounced when the C-terminus + pocket was used as a substrate, whereas no stimulating effect was detected with the N-terminus. As control we used a polypeptide of comparable molecular weight, Penicillium-antifungal peptide, to rule out an unspecific protein effect. Densitometric evaluation of autoradiograms of Figure 4 revealed that HPV16-E7 enhanced acetylation of p130 full-length protein 1.28-fold, acetylation of p130C-terminus + pocket 1.65 -fold, whereas acetylation of p130-N-terminus was unaffected by HPV16-E7 (Supplementary Table 1). Obviously, the E7 protein has a substrate- and site-specific effect on p300 affinity for p130. The well-known binding of histone deacetylases to p130 might not only be due to a targeting role of p130 for histone deacetylases to distinct promoters, but may also reflect binding of the enzyme to exert its catalytic activity on p130 for modulating its functional properties. The functional changes of p130 induced by de/acetylation most likely change its interaction 
properties with other proteins, as known from a number of acetylatable non-histone proteins.

\section{Conflict of interest}

The authors declare no conflict of interest.

\section{Acknowledgements}

We thank A Giordano for providing a p130 plasmid, J Kadonaga for a p300 vector, F Marx-Ladurner for PAF, $G$ Bonn for mass spectrometry facilities and L Huber, A Lusser and M Saeed for valuable discussions. This work was supported by a grant of the Tyrolean Science Foundation to JM.

\section{References}

Brosch G, Loidl P, Graessle S. (2008). Histone modifications and chromatin dynamics: a focus on filamentous fungi. FEMS Microbiol Rev 32: 409-439.

Canhoto AJ, Chestukhin A, Litovchick L, DeCaprio JA. (2000). Phosphorylation of the retinoblastoma-related protein p130 in growth-arrested cells. Oncogene 19: 5116-5122.

Chan HM, Demonacos MK, Smith L, Demonacos C, La Thangue NB. (2001). Acetylation control of the retinoblastoma tumour-suppressor protein. Nat Cell Biol 3: 667-675.

Chestukhin A, Litovchick L, Rudich K, DeCaprio JA. (2002). Nucleocytoplasmic shuttling of p130/RBL2: novel regulatory mechanism. Mol Cell Biol 22: 453-468.

Choi CH, Hiromura M, Usheva A. (2003). Transcription factor IIB acetylates itself to regulate transcription. Nature 424: 965-969.

Classon M, Dyson N. (2001). p107 and p130: versatile proteins with interesting pockets. Exp Cell Res 264: 135-147.

Claudio PP, Tonini T, Giordano A. (2002). The retinoblastoma family: twins or distant cousins? Genome Biol 3: 3012.1-3012.9.

Cobrinik D. (2005). Pocket proteins and cell cycle control. Oncogene 24: 2796-2809.

Du W, Pogoriler J. (2006). Retinoblastoma family genes. Oncogene 25 : 5190-5200.

Felsani A, Mileo AM, Paggi MG. (2006). Retinoblastoma family proteins as key targets of the small DNA virus oncoproteins. Oncogene 25: 5277-5285.

Giacinti C, Giordano A. (2006). RB and cell cycle progression. Oncogene 25: 5220-5227.

$\mathrm{Gu}$ W, Roeder RG. (1997). Activation of p53 sequence-specific DNA binding by acetylation of the p53 C-terminal domain. Cell 90: 595-606.

Hansen K, Farkas T, Lukas J, Holm K, Ronnstrand L, Bartek J. (2001). Phosphorylation-dependent and -indpendent functions of p130 cooperate to evoke a sustained G1 block. EMBO $J \mathbf{2 0}$ : $422-432$.

Hansen K, Lukas J, Holm K, Kjerulff AA, Bartek J. (1999). Dissecting functions of the retinoblastoma tumor suppressor and the related pocket proteins by integrating genetic, cell biology, and electrophoretic techniques. Electrophoresis 20: $372-381$.

Jackson MW, Agarwal MK, Yang J, Bruss P, Uchiumi T, Agarwal ML et al. (2005). p130/p107/p105Rb-dependent transcriptional repression during DNA-damage-induced cell-cycle exit at $\mathrm{G}_{2}$. $J$ Cell Sci 118: 1821-1832.

Litovchick L, Chestukhin A, DeCaprio JA. (2004). Glycogen synthase kinase 3 phosphorylates RBL2/p130 during quiescence. Mol Cell Biol 24: 8970-8980.

Macaluso M, Montanari M, Giordano A. (2006). Rb family proteins as modulators of gene expression and new aspects regarding the

interaction with chromatin remodeling enzymes. Oncogene $\mathbf{2 5}$ 5263-5267.

Mantovani F, Piazza S, Gostissa M, Strano S, Zacchi P, Mantovani R et al. (2004). Pin 1 links the activities of $\mathrm{c}-\mathrm{Abl}$ and $\mathrm{p} 300$ in regulating p73 function. Mol Cell 14: 625-636.

Mayol X, Garriga J, Grana X. (1996). G ${ }_{1}$ cyclin/CDK-independent phosphorylation and accumulation of p130 during the transition from $G_{1}$ to $G_{0}$ lead to its association with E2F-4. Oncogene 13: 237-246.

Meraner J, Lechner M, Loidl A, Goralik-Schramel M, Voit R, Grummt I et al. (2006). Acetylation of UBF changes during the cell cycle and regulates the interaction of UBF with RNA polymerase I. Nucl Acids Res 34: 1798-1806.

Nelson CJ, Santos-Rosa H, Kouzarides T. (2006). Proline isomerization of histone $\mathrm{H} 3$ regulates lysine methylation and gene expression. Cell 126: 905-916.

Nguyen DX, Baglia LA, Huang SM, Baker CM, McCance DJ. (2004) Acetylation regulates the differentiation-specific functions of the retinoblastoma protein. EMBO J 23: 1609-1618.

Popov B, Chang LS, Serikov V. (2005). Cell-cycle-related transformation of the E2F4-p130 repressor complex. Biochem Biophys Res Commun 336: 762-769.

Roman A. (2006). The human papilomavirus E7 protein shines a spotlight on the pRB family member, p130. Cell Cycle 5: 567-568.

Sims RJ, Reinberg D. (2008). Is there a code embedded in proteins that is based on post-translational modifications? Nat Rev Mol Cell Biol 9: $1-6$.

Tedesco D, Lukas J, Reed SI. (2002). The pRb-related protein p130 is regulated by phosphorylation-dependent proteolysis via the proteinubiquitin ligase SCF (Skp2). Genes Dev 16: 2946-2957.

Vervoorts J, Lüscher-Firzlaff JM, Rottmann S, Lilischkis R, Walsemann G, Dohmann K et al. (2003). Stimulation of c-Myc transcriptional activity and acetylation by recruitment of the cofactor CBP. EMBO Rep 4: 484-490.

White MK, Khalili K. (2006). Interaction of retinoblastoma protein family members with large T-antigen of primate polyomaviruses. Oncogene 25: 5286-5293.

Zhang Y, Li N, Caron C, Matthias D, Hess S, Khochbin S et al (2003). HDAC-6 interacts with and deacetylates tubulin and microtubules in vivo. EMBO J 22: 1168-1179.

OMMERIEHIS RESE

This work is licensed under the Creative Commons Attribution-NonCommercial-No Derivative

Works 3.0 Unported License. To view a copy of this license, visit http://creativecommons.org/licenses/by-nc-nd/3.0/

Supplementary Information accompanies the paper on the Oncogene website (http://www.nature.com/onc) 